\title{
Office wall color influence on subjective response in patients with chronic heart failure
}

\author{
Aida Botonjic Karahusic ${ }^{1 *}$, Nedim Begic ${ }^{2}$, Edin Begic ${ }^{3,4}$, Sabina Kusljugic ${ }^{5}$, Damir Secic ${ }^{6}$ \\ ${ }^{1}$ Department for Architectural Structures and Building Technologies, Faculty of Architecture, University of Sarajevo, Bosnia \\ ${ }^{2}$ Department of Cardiology, Pediatric Clinic, Clinical Center University of Sarajevo, Bosnia \\ ${ }^{3}$ Department of Cardiology, General Hospital "Prim.Dr. Abdulah Nakas", Bosnia \\ ${ }_{5}^{4}$ Department of Pharmacology, Sarajevo Medical School, Sarajevo School of Science and Technology, Bosnia \\ ${ }^{5}$ Faculty of Medicine, University of Tuzla, Bosnia \\ ${ }^{6}$ Department for Pathophysiology, Faculty of Medicine, University of Sarajevo, Bosnia
}

*Corresponding author: aidab@af.unsa.ba

(C) The Author(s)

2020.

Published by ARDA.

\begin{abstract}
Heart failure is defined as a clinical state, which occurs as a result of structural or functional damage of ventricle with consequential blood hypo perfusion of organs (reduced stroke volume of heart muscle and/or increased intracardial pressure in rest or during work activity).

Therapeutical monitoring of patient is imperative, and it includes assessment of objective and subjective state of patient, which is often guide for optimizing of pharmacological treatment. The aim of paper is assessment of the room wall color influence as one of the factor for optimizing the therapeutic modality of patients with diagnosis of heart failure. Findings suggest that the influence of color in environment can be neutral, enabling or disabling, and this is the aspect of treatment that needs to be investigated further in future.
\end{abstract}

Keywords: Office wall color, Heart failure, Treatment, Software, Tools

\section{Introduction}

Heart failure (HF) represent global problem with at least 26 million patients around the globe [1,2]. It is defined as a clinical state which occurs as a result of structural or functional damage of ventricle with consequential blood hypo perfusion of organs (reduced stroke volume of heart muscle and/or increased intracardial pressure in rest or during work activity) [3].

It can be classified on acute or chronic, systolic and diastolic, and in relation to ejection fraction of left ventricle on heart failure with reduced ejection fraction ( $\mathrm{HFrEF}$ ), heart failure with preserved ejection fraction (HFpEF) and heart failure with mid-range ejection fraction. The diagnosis of HF is established with adequate medical anamnesis, physical status, laboratory examination, and through use of noninvasive and invasive diagnostic tools. Prognosis of HF is not optimistic, and it is determined by many variables such as age, clinical condition, cause of heart failure and degree of HF [3]. Prognosis is better when the treatment of the cause of $\mathrm{HF}$ is possible. According to researches, during the first year of diagnosis establishment risk of death is around $35 \%$, while $70 \%$ of patients with this diagnosis die in 10 year period since the diagnosis established $[3,4,5]$. Rehabilitation of patients with HF has an important role in increasing the quality and length of life. In therapy, we use angiotensin-converting-enzyme inhibitors (ACE-inhibitors) or angiotensin receptor blockers (ARB), with beta blockers, mineralocorticoid-receptor antagonists and diuretic therapy [3]. Considering the 
etiology of HF occurrence the place in treatment also have nitric oxide derivatives, and metabolic agents (trimetazidine, ranolazine) [3]. Frequent medical examinations are very important, during which clinical state and subjective feeling are controlled (health-related quality of life questionnaire; EuroQoL-5D (EQ-5D), European Heart Failure Self-Care Behavior Scale (EHFScBS), Chronic Heart Failure Questionnaire (CHQ), Minnesota Living with Heart Failure Questionnaire (MLHF-Q)) [3,5,6]. Subjecting feeling related to the state is often associated with mental component and his value sometimes can be deceiving, which can make therapeutic modality optimizing rather complicated.

The aim of this paper is to assess the influence of color as a factor of optimizing the therapeutic modality of patients with HF diagnosis. It also explores the communication between situated patient and the space (office) where an important part of communication takes place through color, and that visual stimuli is later followed by a certain reaction due to the visual perception.

\subsection{Determinants of the design of the constructed space on the patients' health}

The color in everyday life has a multiple meaning, and every attempt of defining that phenomenon, have resulted in incomplete and heterogeneous definitions that still don't give as a clear view on the color phenomenon (Figure 1) [7]. We consider the color as a reaction of photoreceptors in our eye on external stimulus - light ray. By entering in eye the light ray breaks like in prism and disperses in the color spectrum. [8]

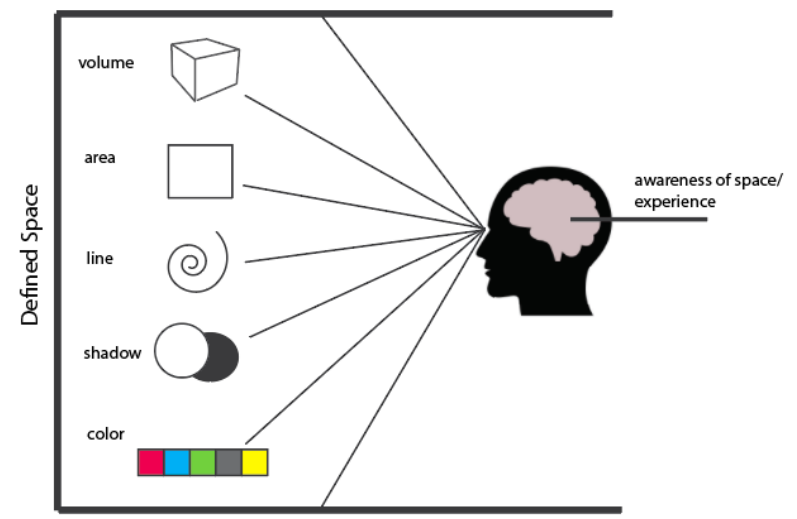

Figure 1. Perception of the space - relation of consciousness and experience with elements: volume, surface, lines, shadows and colors [9]

It is defined as one of the dominant sensory characteristics and based on that the viewer has the perception of the world around him and defines certain judgment, which is one of the key elements for space design. Achieving the positive relation of colors inside the defined space is key for forming the positive picture for the viewer. During this process the brain process what it is about, perceiving the color on objective and subjective level, and during the visual communication with surfaces, lines, lightning, contrasts and other information and stimuli, created the individual process of judgment. In the last few decades empirical observations and scientific studies were stated about the reaction of the man inside defined environment, on stimulus that are based on sensory perception of colors. The studies that were conducted to state different effects of colors on man, in most of the cases were multidisciplinary characteristics and the include field of psychology, architecture and urbanism, neuropsychology, visual ergonomics and psycho-motoric. It is established that human response on color is integrative, both psychological and physiological. It has been discovered that every color has certain psychological effect on human. Red is perhaps the most dominant and most dynamic color. Energy of this color effects powerfully on the plant growth. It has been discovered that it accelerates development of the lower animals, increases hormonal and sexual activity and wound healing. The same case is with yellow color, which provokes the most intense straining of the eyes than any other color in color 
spectrum. Blue and green color have tendency to provoke cheerful emotions at viewer, and are pleasant to the eye. Straining of the eyes is absent during visual contact with blue and green color. The model indicates that the personal reaction to the color is basically intertwined experience, that includes series of psychological and physiological influences, such as: biologically unavoidable reactions, memories from unconscious, symbolic of conscious, cultural influences and mannerisms, trend influences, fashion and style, and personal relationship of individual. Mahnke has divided human experience related to colors in six basic interconnected levels (Figure 2) [10].

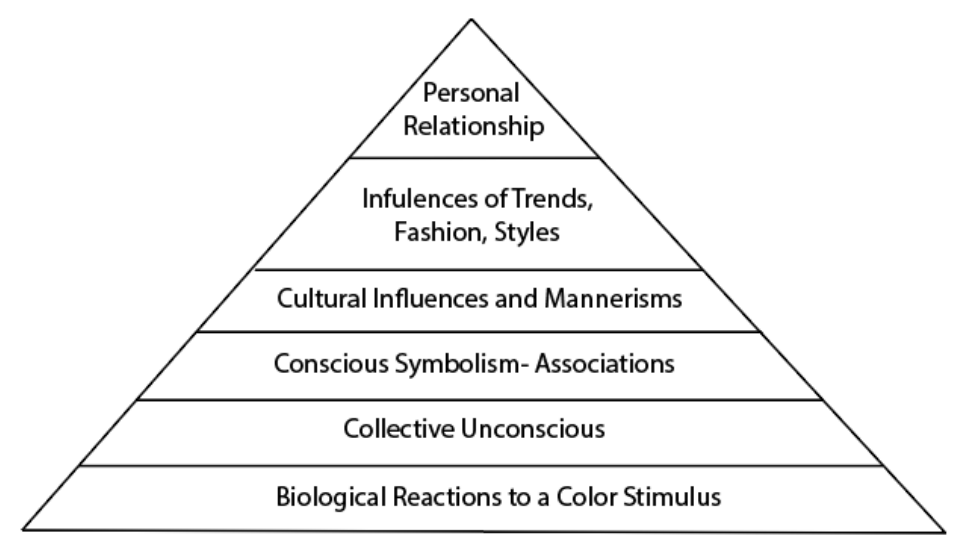

Figure 2. Color Experience Pyramid [10,11]

Health is a state of a complete physical, mental and social well-being and not merely the absence of disease or infirmity [12]. Color and color design can have an affect on cognitive function, behavioral responses and can induce different response on some stress situations $[13,14,15]$. Human determine color in environment as a positive distraction or a stressor, depending on the individual's feelings or experiences. Color meaning is influenced by people's experience, especially by shared experience in the cultural context. Mental health distractions caused by color can negatively impact on physical health, leading to an increased risk on some medical conditions. Many researches stated that there is influence of the color on humans' mental health (disorientation, tiredness, stress) and physical health (heart pressure disorder, appetite, sleep disorder) $[10,11,13]$. By using certain colors, a positive environmental effect on the mental and thus the clinical condition of the patients can be achieved $[10,11,15]$.

\section{Material and methods}

The computer models of the cardiology offices were made in Adobe Illustrator CC 2020 software (Adobe Inc., San Jose, California, United States of America) and Adobe Photoshop (Adobe Inc., San Jose, California, United States of America). The study had a prospective character. Office modalities were presented to patients diagnosed with HF. The study included 75 patients with ejection fraction of left ventricle (EFLV) 25-40\%, and which did not have an episode of acute heart failure in the last six months. Patients were asked the following questions:

1. Which office on the picture seems most appropriate for physical examination? (Figure 3)

2. Do you have a problem with the subjective acceptance of the symptoms of your illness?

3. Do you think that the color of the office can have an effect on your subjective feeling of clinical symptoms?

4. Which treadmill would you choose to perform exercise on it? (Figure 4) 
Results are presented by number of cases and percentages. Chi-squared test $\left(\chi^{2}\right)$ was used to compare the answers. The Microsoft Excel (version 11.0, Microsoft Corporation, Redmond, WA, United States of America) was used to process the questionnaire data.

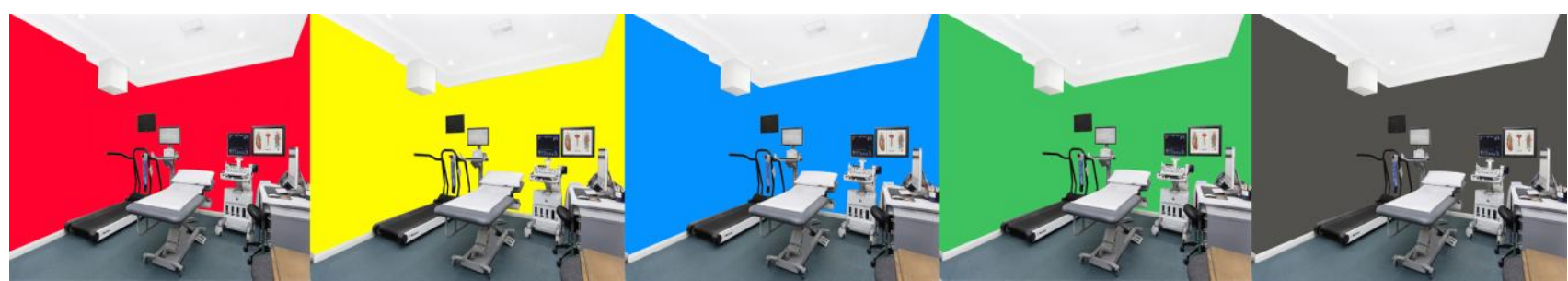

Figure 3. Color modalities of cardiology office
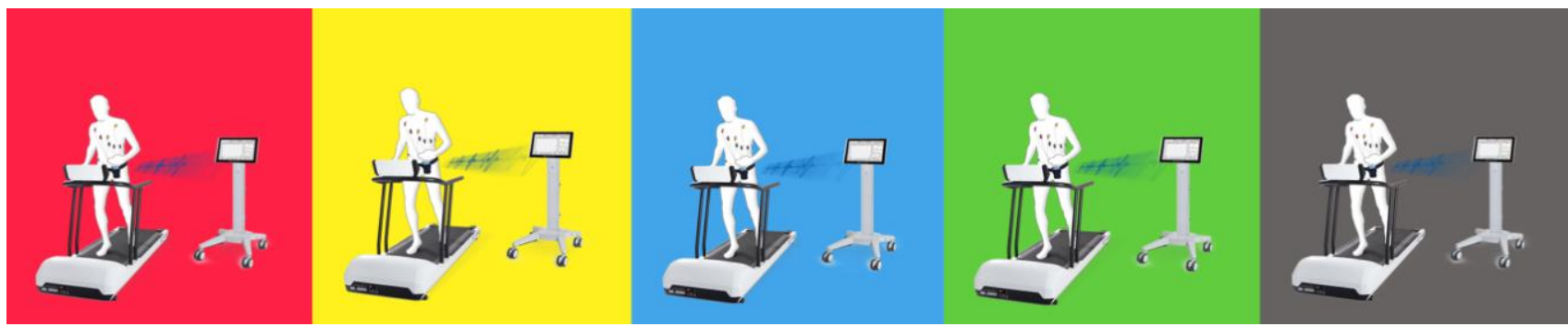

Figure 4. Color modalities of treadmill background

\section{Results}

When asked "Which office on the picture seems most appropriate for physical examination?" 39 (52\%) respondents chose blue color (Table 1). When asked, "Do you have a problem with the subjective acceptance of the symptoms of your illness", 59 (78.6\%) respondents had problems with the subjective feeling of acceptance of symptoms. On question "Do you think that the color of the office may have an effect on your subjective feeling of clinical symptoms", $30(40 \%)$ of the respondents thought that the color of the office walls could have an effect on the subjective feeling about the clinical symptoms during physical examination. When asked, "Which treadmill would you choose to perform exercise on it?", 47 (62.6\%) respondents said they would run on a blue background treadmill (Table 2).

Table 1. Respondent's response to opinion on the background of the office

\begin{tabular}{|l|l|l|l|l|l|}
\hline & red & blue & yellow & green & grey \\
\hline $\begin{array}{l}\text { Which office on the picture } \\
\text { seems most appropriate for } \\
\text { physical examination? }\end{array}$ & $\begin{array}{l}16 \\
(21.3 \%)\end{array}$ & $39(52 \%)$ & $7(9.3 \%)$ & $9(12 \%)$ & $4(5.3 \%)$ \\
\hline
\end{tabular}
$\chi^{2}=50.6 ; \mathrm{p}<0.001$

Table 2. Respondent's response to opinion on the background of the treadmill

\begin{tabular}{|l|l|l|l|l|l|}
\hline & red & blue & yellow & green & grey \\
\hline $\begin{array}{l}\text { Which treadmill would you } \\
\text { choose to perform exercise on it? }\end{array}$ & $(22.6 \%)$ & $\begin{array}{l}77 \\
(62.6 \%)\end{array}$ & $7(9.3 \%)$ & $5(6.6 \%)$ & $0(0 \%)$ \\
\hline
\end{tabular}

$\chi^{2}=50.6 ; \mathrm{p}<0.001$ 


\section{Discussion}

In 1810, Goethe gave his theory of color as an answer to emotion [13]. Out of total number, $78.6 \%$ of the respondents in this study had difficulties to accept their disease. In this research, $40 \%$ of the respondents thought that the color would have an effect on them during the clinical examination. For most respondents, blue is the color of their choice. Blue light has been shown to increase subjective alertness and performance on attention-based tasks $[14,15]$.

Holzman stated that blue has the greatest effect on body restart to daily rhythm, which decreases in green environment and that the smallest effect has a red color [16]. The same author stated that blue has an effect in circadian rhythm, that blue waves can be useful for the treatment of premenstrual depression and bulimia, as well as for treatment of anxiety [16]. He also stated that blue in artificial light can have an effect in prevention of cancer and cardiovascular disease, reducing sleep disturbances, suppressing melatonin for about twice longer then green, that it can be useful for raising body temperature and heart rate, to reduce the need for sleep and has an effect on the memory process (stimulates activity in the left frontal and parietal cortices) [16].

Tests that assess a patient's subjective feeling are an integral part of daily clinical work of an internal medicine specialist or cardiologist with patients diagnosed with HF. All of the above indicates that we should think about environment of the patient which could be an additional factor in optimizing of therapeutic modality.

\section{Conclusions}

The patient's subjective feelings should be part of the evaluation and optimization of the therapeutic modality of patients diagnosed with HF. Color, should be part of the strategy of organizing office for patients with HF, as it could additionally affect better and more optimized treatment. Positive psychology must be part of the multidisciplinary modality of this type of patients.

\section{References}

[1] G. Savarese, L.H. Lund, "Global Public Health Burden of Heart Failure", Card Fail Rev, Vol. 3, pp. 711, 2017.

[2] E. Braunwald, "The war against heart failure: the Lancet lecture", Lancet, Vol. 385, pp. 812-824, 2015.

[3] P. Ponikowski, A.A.Voors, S.D. Anker, Authors/Task Force Members, Document Reviewers, "2016 ESC Guidelines for the diagnosis and treatment of acute and chronic heart failure: The Task Force for the diagnosis and treatment of acute and chronic heart failure of the European Society of Cardiology (ESC)", European Journal of Heart Failure, Vol. 18(8). pp 891-975, 2016.

[4] E. Begic, Z. Begic, N. Naser, "Clinical Course and Treatment of Dilated Cardiomyopathy During Twenty Years of Follow-up", Med Arch., Vol. 72(1), pp 68 - 70, 2018.

[5] W.C. Little, M.R. Zile, "HFpEF: Cardiovascular abnormalities not just comorbidities", Circ Heart Fail. Vol. 5, pp $669-671,2012$.

[6] "Questionnaires Used for the Assessment of Quality of Life in Patients with Cardiovascular Diseases with Relevant References". URL:https://link.springer.com/content/pdf/bbm\%3A978-88-470-27695\%2F1.pdf (retrieved on February 20, 2020).

[7] W. Alberts, M.T. van der Geest, "Color matters: color as trustworthiness cue in websites", Tech. Comm. Vol. 58, pp 149-160, 2011.

[8] F. Birren, „Color Psychology and Color Therapy: A Factual Study of the Influence of Color on Human Life“. Martino Fine Books, Connecticut, United States of America. 10-40, 1961.

[9] R. Tofle, Brent,B., Schwarz, Y.D. Yoon, A. Max-Royale, "Color in healthcare enviroments". Coalition for HealthEnvironments Research (CHER), San Francisco, United States of America. 38-43. 
[10] López-Tarruella J, Llinares Millán C, Serra Lluch J, Iñarra Abad S, Wijk H, "Influence of Color in a Lactation Room on Users' Affective Impressions and Preferences" HERD. Vol. 12(2), pp 55-70, 2019.

[11] F.H. Mahnke, "Color, Environment, and Human Response: An Interdisciplinary Understanding of Color and Its Use as a Beneficial Element in the Design of the Architectural Environment" Van Nostrand Reinhold, New York, 1-234, 1996.

[12] "Health definition", URL: http://apps.who.int/gb/bd/pdf files/BD 49th-en.pdf\#page=1 (retrieved on March 10, 2020).

[13] S.W. Lockley, E.E. Evans, F.A. Scheer, C.G. Brainard, C.A. Czeisler, D. Aeschbach, "Shortwavelength sensitivity for the direct effects of light on alertness, vigilance, and the waking electroencephalogram in humans", Sleep, Vol. 29, pp 161-168, 2006.

[14] A.J. Elliot, "Color and psychological functioning: a review of theoretical and empirical work", Front Psychol.Vol. 6, pp 368, 2015.

[15] D.C. Holzman, "What's in a color? The unique human health effect of blue light", Environ Health Perspect, Vol. 118, pp A22-A27, 2010. 